\title{
Water-Energy-Food Nexus: Rethinking the Integrated Resources Management and Modeling
}

\author{
Amin Elshorbagy \\ Department of Civil, Geological, and Environmental Engineering \& Global Institute for Water Security, \\ University of Saskatchewan, Saskatoon, Canada
}

\begin{abstract}
Sustainable development requires the effective management of essential life-supporting resources-water, energy, and food (WEF) - that are under pressure due to socio-economic development and climate change. Addressing WEF security challenges requires an understanding of the complex behavior of the WEF nexus, which often integrates human systems (e.g. economy, energy, land use) and natural systems (e.g. hydrology, biology,) in the same framework. For long time, we have worked with integrated resource (e.g. water) management (IWRM), focusing on the impact of water resources use on the environment and economy. The community has been building planning and management models for simulation and optimization of systems, conceptualized using the IWRM concept. However, IWRM places water sectors in the center of the decision-making sphere; driving the integrated system and dictating the rules in a singleboundary system based on scenarios of water availability. This is not perfectly realistic as other basic human needs, such as energy and food, are other boundaries and driving forces. Therefore, the multi-centric, multiple boundaries conceptualization of water-energy-food nexus is presented as an alternative and more realistic approach to integrated resource management.

In this keynote talk, the WEF nexus approach is presented with quantitative demonstration from the Canadian Prairies. Zooming further down on the province of Saskatchewan, the recently developed WEF-Sask model is presented. The model is used to investigate the impacts of socioeconomic and hydroclimatic variables on WEF systems and to identify the trade-offs and synergies across the WEF nexus under various decisions and strategies. This model incorporates both production (supply) and demand sides of the water, energy, and food systems into a single modeling framework, with a key objective to quantify the interactions between WEF sectors. WEF-Sask model allows for systematic analysis of issues pertinent to coupled human and natural systems. The model results suggest that the agriculture (food and feed) sector and irrigation water use are highly sensitive to climate change, while socio-economic factors significantly impact the energy sector, water demand in municipal and industrial sectors, and GHG emissions. Population, GDP, crude oil reserve, air temperature, and precipitation are influential factors for total water demand. Total energy demand is highly sensitive to population and GDP, while total energy production is more sensitive to crude oil reserves and moderately sensitive to natural gas reserves. The model helps address the dynamics of sustainability challenges, trade-offs, and synergies in the WEF systems under drivers, such as climate change, population growth, economic development, and adaptation and resilience strategies and policies.
\end{abstract}

